\title{
VIPergic neurons of the infralimbic and prelimbic cortices control palatable food intake through separate cognitive pathways
}

\author{
Brandon A. Newmyer, ${ }^{1}$ Ciarra M. Whindleton, ${ }^{1}$ Peter M. Klein, ${ }^{1}$ Mark P. Beenhakker, ${ }^{1}$ \\ Marieke K. Jones, ${ }^{2}$ and Michael M. Scott ${ }^{1}$ \\ 'Department of Pharmacology, and ${ }^{2}$ Health Sciences Library, University of Virginia, Charlottesville, Virginia, USA.
}

\begin{abstract}
The prefrontal cortex controls food reward seeking and ingestion, playing important roles in directing attention, regulating motivation toward reward pursuit, and assigning reward salience and value. The cell types that mediate these behavioral functions, however, are not well described. We report here that optogenetic activation of vasoactive intestinal peptide-expressing (VIP-expressing) interneurons in both the infralimbic (IL) and prelimbic (PL) divisions of the medial prefrontal cortex in mice is sufficient to reduce acute, binge-like intake of high-calorie palatable food in the absence of any effect on low-calorie rodent chow intake in the sated animal. In addition, we discovered that the behavioral mechanisms associated with these changes in feeding differed between animals that underwent either IL or PL VIPergic stimulation. Although IL VIP neurons showed the ability to reduce palatable food intake, this effect was dependent upon the novelty and relative value of the food source. In addition, IL VIP neuron activation significantly reduced novel object and novel social investigative behavior. Activation of PL VIP neurons, however, produced a reduction in high-calorie palatable food intake that was independent of food novelty. Neither IL nor PL VIP excitation changed motivation to obtain food reward. Our data show how neurochemically defined populations of cortical interneurons can regulate specific aspects of food reward-driven behavior, resulting in a selective reduction in intake of highly valued food.
\end{abstract}

Conflict of interest: The authors have declared that no conflict of interest exists.

Copyright: () 2019 American Society for Clinical Investigation

Submitted: November 19, 2018 Accepted: March 27, 2019

Published: May 2, 2019.

Reference information: /CI Insight. 2019;4(9):e126283. https://doi. org/10.1172/jii.insight.126283.

\section{Introduction}

Excessive palatable food consumption is a significant contributor to the development of obesity. Although the ability of neuronal mechanisms to override the homeostatic control of food intake during periods of energy abundance can lead to overeating $(1,2)$, the identities of specific cortical cell types that can promote changes in feeding behavior are not well described.

The medial prefrontal cortex (mPFC), encompassing the anterior cingulate, infralimbic (IL), and prelimbic (PL) cortices, has been implicated in the control of a myriad of functions, including the attribution of salience (3), the regulation of binge food intake $(4,5)$, reward prediction error signaling $(6,7)$, and the drive to expend effort to obtain reward (8-10). Prior studies within this region have shown how dopamine (11) and opioid (12-14) action can regulate feeding on both rodent chow and palatable, energy-dense food. However, little is known of how specific neurochemically defined frontal cortical neuronal populations respond to these and other signals to produce a change in either the consumption of or motivation to obtain food. Furthermore, although prior studies have shown how the IL and PL cortices play complementary roles in the regulation of behavioral expression (15-17), the importance of defined subtypes of neurons in either the IL or PL cortex in the control of food intake has yet to be investigated.

In both the IL and PL subdivisions of the $\mathrm{MPFC}, \mathrm{GABAergic} /$ vasoactive intestinal peptide-expressing (VIPergic) interneurons are well positioned to regulate feeding behavior. Serving as a convergence point for extracortical glutamatergic (18), serotonergic (19), and cholinergic (19-21) inputs that have previously been implicated in the control of food intake, VIP cells become active following reward presentation (22), which suggests they act as a feedback signal that conveys a reward prediction error (7). 
To investigate the sufficiency of VIPergic interneurons in the regulation of food intake, we used a stabilized step-function opsin (SSFO) (23) to produce VIPergic depolarization and subsequent neuronal activation in either the IL or PL subdivisions of the mouse mPFC. Below, we describe how VIPergic neuronal stimulation in either the IL or PL of sated animals can selectively reduce consumption of palatable food in the absence of any effect on rodent chow consumption or on the drive to work to obtain a palatable food reward. Surprisingly, we also discovered that the ability of IL VIPergic neurons to suppress food intake was likely dependent upon the novelty and relative value of the food source. PL VIPergic neurons, however, modulate feeding behavior of both novel palatable food and familiar food with high perceived value. Thus, our work describes for the first time to our knowledge a neuronal cell type in the cortex capable of selectively reducing the consumption of palatable food while sparing normal chow consumption in sated animals, through the engagement of 2 behavioral mechanisms.

\section{Results}

Selective activation of the IL and PL cortices by SSFO expression in VIPergic neurons. Because VIP neurons show both burst firing and regular firing properties in response to depolarization (24), we reasoned that to stimulate VIP cells at a specific firing frequency might not reproduce the most physiologically relevant activation of this cell type. Thus, we chose to use an SSFO to produce neuronal depolarization in VIP neurons without dictating a specific firing frequency. After injecting a Cre recombinase-dependent SSFO into either the IL (IL SSFO, Figure 1A) or PL (PL SSFO, Figure 1B) of heterozygous mice expressing Cre recombinase from the VIP locus, optical fibers were unilaterally inserted to allow for light-driven neuronal activation.

Following a 5-second light pulse, in IL SSFO mice we observed significant neuronal activation by c-Fos

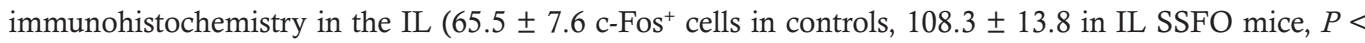
0.05, quantified in Figure 2A, visualized in Figure 2, B and C for IL controls and SSFO mice, respectively)

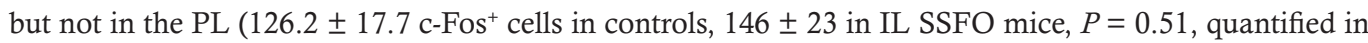
Figure 2D, visualized in Figure 2, E and F for IL controls and SSFO mice, respectively). Similarly, following a 5-second light pulse in PL SSFO mice, we observed significant neuronal activation by c-Fos immunohistochemistry in the PL $\left(131 \pm 7\right.$ c-Fos $^{+}$cells in controls, $181.6 \pm 14.5$ in PL SSFO mice, $P<0.05$, Figure $3 \mathrm{D}$, visualized in Figure 3, B and C for controls and PL SSFO mice, respectively) but not the IL (98.9 \pm 7.7 c-Fos+ cells in controls, $78.6 \pm 7$ in PL SSFO mice, $P=0.08$, Figure $3 \mathrm{~A}$, visualized in Figure $3, \mathrm{E}$ and $\mathrm{F}$ for controls and PL SSFO mice, respectively).

Although we did not directly test whether VIPergic neurons showed activation following light exposure, prior studies have validated both the Cre-dependent expression of the SSFO vector (23) and the selective expression of Cre recombinase in VIPergic neurons of the VIP-IRES-Cre mouse line (7). Thus, we subsequently used enhanced yellow fluorescent protein (EYFP) expression from the SSFO vector as a proxy for VIP expression in our work.

Importantly, when we stimulated with SSFO in the IL, the degree of presumptive VIP neuron activation was significantly greater in the IL than when SSFO stimulation occurred in the PL brain region (after light stimulation, $81.8 \% \pm 3.9 \%$ total $\mathrm{EYFP}^{+}$cells also c-Fos ${ }^{+}$in the IL of IL SSFO mice, $35.7 \% \pm 6.6 \%$ in the IL of PL SSFO mice, $P<0.001$, Figure 4 , A-C). Results were similar when we targeted the PL, with lower activation of presumptive VIPergic neurons in the neighboring IL brain region being observed (after light stimulation, $78.5 \% \pm 4 \%$ total $\mathrm{EYFP}^{+}$cells also c-Fos ${ }^{+}$in the PL of PL SSFO mice, $51.4 \% \pm 4.6 \%$ in the PL of IL SSFO mice, $P<0.001$, Figure $4, \mathrm{D}-\mathrm{F}$ ).

These data demonstrate both our ability to selectively target either the IL or PL as well as how VIP neuron stimulation in either brain region can produce spatially localized neuronal excitation. Our data also agree with prior work that showed an increase in c-Fos expression following VIPergic activation in the cortex (7) and suggest that the result of VIPergic excitation is indeed neuronal disinhibition. In addition to investigating the actions of SSFO in vivo, we performed slice electrophysiology on brain sections expressing SSFO and a yellow fluorescent protein (YFP) reporter in VIPergic neurons. Following a 5-second light pulse, VIP neurons showed a significant increase in resting membrane potential in the current clamp, confirming the excitatory actions of SSFO in this cell type (Figure 4G, representative trace. Mean change in depolarization $4.467 \pm 1.500 \mathrm{mV}, n=3$ neurons).

Activation of both IL and PL VIP subpopulations decreases intake of highly valued food but has no effect on motivation to obtain palatable food. To elucidate roles for IL and PL VIP neurons in the binge-like, acute overconsumption of food, we stimulated IL or PL VIPergic neurons with a 5-second light pulse train, then, in 2 experiments, allowed mice free access to either standard chow or to a highly palatable high-calorie diet 

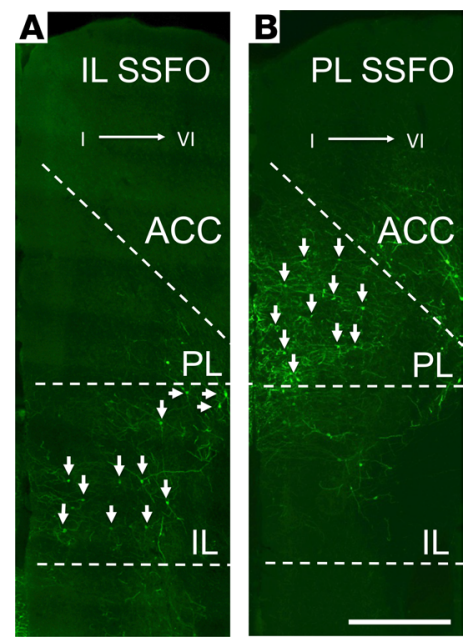

Figure 1. SSFO expression in the PL and IL. AAV-driven expression of SSFO was selective for the IL (A) and PL (B). Arrowheads indicate SSFO-expressing neurons in representative images of animals with AAV injections targeted to either the IL or PL cortex. Expression of SSFO was observed in all study animals between $+1.7 \mathrm{~mm}$ and $+1.98 \mathrm{~mm}$ rostral of the bregma, corresponding to panels 17 to 19 in Paxinos and Franklin (54). Relative position of cortical layers I $\rightarrow \mathrm{VI}$ indicates orientation of the coronal slice. Scale bar: $500 \mu \mathrm{m}$.

(HCD1). Although neither IL nor PL VIPergic stimulation affected standard chow intake at the start of the animal's light cycle, circadian time 0 (CT0, Figure 5A), both IL and PL VIPergic stimulation were sufficient to reduce intake of the energy-dense HCD1 at 30 minutes after stimulation $(3.02 \pm 0.3 \mathrm{kcal}$ for IL SSFO controls, $1.9 \pm 0.26 \mathrm{kcal}$ for IL SSFO, $P<0.01 ; 4.3 \pm 0.16 \mathrm{kcal}$ for PL SSFO controls, $3.3 \pm 0.34 \mathrm{kcal}$ for PL SSFO, $P<0.05$, Figure 5C) relative to sham-stimulated littermate controls. We hypothesized that the low value of standard chow in sated mice at CT0 (10) may have masked the ability of VIPergic stimulation to reduce feeding. Thus, we subsequently repeated our chow intake study at the onset of subjective night (CT12), when mice show an enhanced drive to feed, and again at CT0 following an 18-hour overnight fast. Although we found that control mice consumed more food at CT12 than CT0, again neither IL nor PL VIP stimulation affected standard rodent chow intake (Figure 5B). However, following a fast to further increase the relative value of chow, stimulation of both PL and IL VIPergic neurons reduced chow intake $(2.199 \pm$ $0.11 \mathrm{kcal}$ for IL SSFO controls, $1.80 \pm 0.091 \mathrm{kcal}$ for IL SSFO, $P<0.05 ; 2.129 \pm 0.068 \mathrm{kcal}$ for PL SSFO controls, $1.64 \pm 0.145 \mathrm{kcal}$ for PL SSFO, $P<0.05$, Figure $5 \mathrm{D}$ ), suggesting that this cell type can regulate feeding when the value of the food source changes.

In addition to the regulation of food intake, the frontal cortex has also been shown to regulate the motivation to obtain food reward $(8,10,25)$. Thus, we investigated whether VIPergic stimulation could also affect the drive to work for food reward using a progressive-ratio operant conditioning paradigm. Unlike the observed effect on palatable food intake, no changes in progressive-ratio operant breakpoint or time to completion of the initial fixed ratios (FRs) in the progressive-ratio paradigm were observed following either PL or IL VIPergic stimulation (Figure 5, E-G). For PL (in seconds): FR5 control $46.57 \pm 12.31$, FR5 SSFO $114 \pm 23.3, P=0.54$; FR10 control $45.85 \pm 10.21$, FR10 SSFO $55.66 \pm 11.69, P=0.92$; FR20 control $179.71 \pm 69.01$, FR20 SSFO $82 \pm 15.58, P=0.35$; FR30 control $192.43 \pm 82.75$, FR30 SSFO $334 \pm 114$ $P=0.56$. For IL (in seconds): FR5 control $47.6 \pm 16.82$, FR5 SSFO $168 \pm 63.34, P=0.159$; FR10 control $59.33 \pm 15.2$, FR10 SSFO 58.16 $\pm 8.0, P=0.94 ;$ FR20 control $53.5 \pm 11.59$, FR20 SSFO $74.83 \pm 10.6, P=$ 0.22 ; FR30 control $76.5 \pm 11$, FR30 SSFO $114.7 \pm 37.86, P=0.39$.

Taken together, these results demonstrate that both IL and PL VIPergic neurons are sufficient to drive a reduction in high-value food intake while sparing effects on the motivation to obtain food reward.

The ability of IL but not PL VIP neuron activation to reduce palatable food intake depends on food novelty. Novelty and salience are both important factors driving pursuit of both natural and drug rewards (26-28). In fact, the availability of a wide variety of energy-dense foods has been suggested to play an important role in driving overeating, preventing the development of sensory-specific satiety $(29,30)$. To examine whether the observed ability of $\mathrm{mPFC}$ VIPergic neurons to reduce food intake was dependent upon the novelty of the food stimulus, we performed an experiment in which mice that underwent our initial food intake evaluation (Figure 5C) were 


\section{IL SSFO Stimulation}
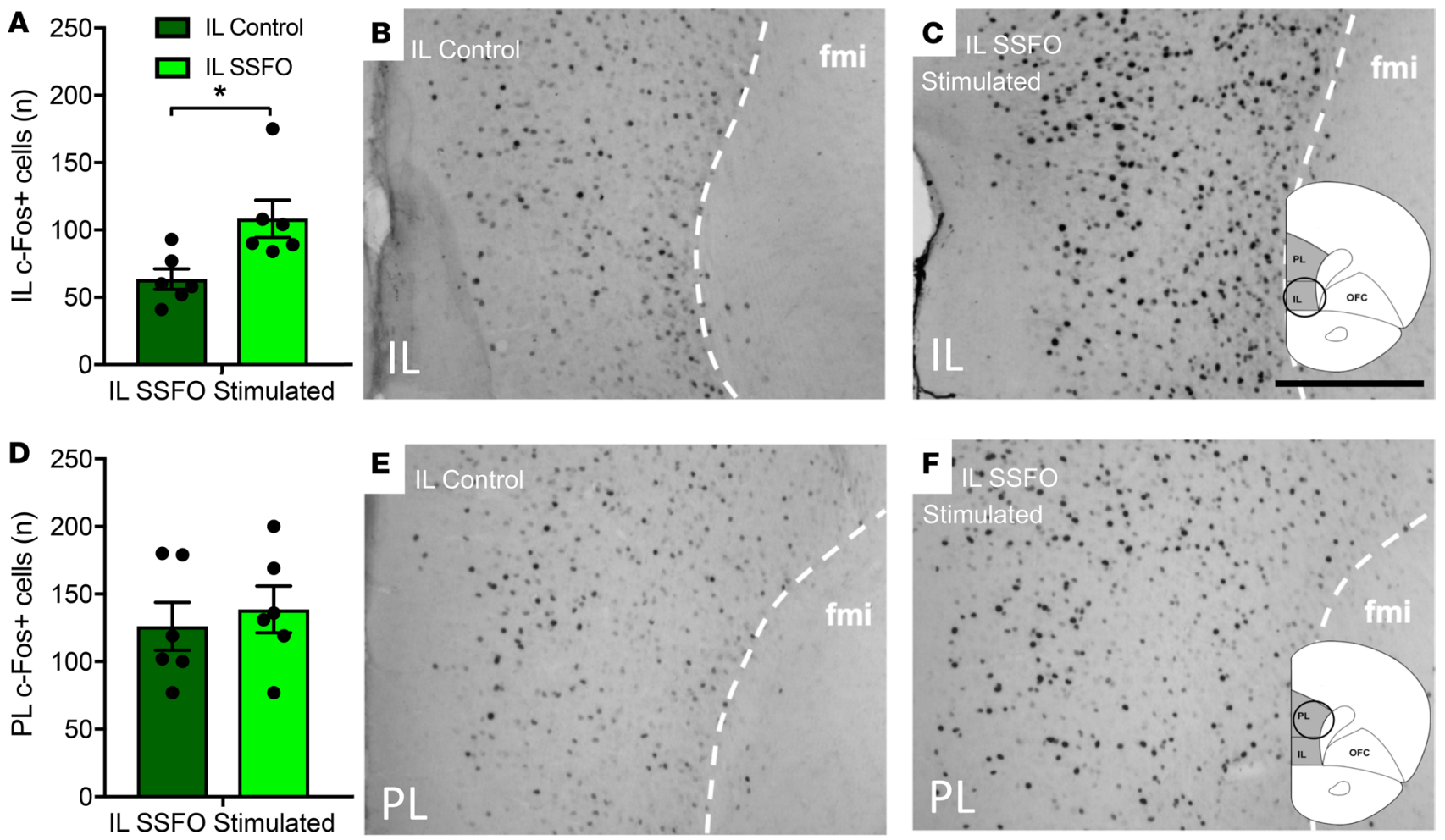

Figure 2. SSFO stimulation of IL VIPergic neurons produces a selective increase in c-Fos expression throughout the IL. IL SSFO stimulation results in an increase in c-Fos expression (C) when compared with controls (B) quantified in (A). IL SSFO stimulation does not result in an increase in c-Fos expression in the PL, when stimulated animals (F) are compared with controls (E) quantified in (D). Scale bar: $200 \mu \mathrm{m}$. Fmi, forceps minor corpus callosum. ${ }^{*} P<0.05$, unpaired 2-tailed $t$ test. $6 \mathrm{IL}$ control and 6 IL SSFO mice (A and D) were used for analysis.

retested using the same diet (HCD1) after either IL or PL VIP stimulation (Figure 6, A and C, for IL and PL, respectively). Interestingly, while IL VIP stimulation failed to affect palatable food intake following the animal's second exposure to the food (2-way repeated measures ANOVA, $F_{1,9}=9.434, P=0.0133,2.84 \pm 0.3 \mathrm{kcal}$ for IL SSFO control during second exposure, $2.42 \pm 0.6 \mathrm{kcal}$ for IL SSFO second exposure; $P=0.19)$. PL VIP stimulation significantly reduced HCD1 consumption during both the initial and second exposures (2-way repeated measures ANOVA, $F_{1,14}=8.785, P=0.0103,4.08 \pm 0.23 \mathrm{kcal}$ for PL SSFO control during second exposure, 3.25 $\pm 0.26 \mathrm{kcal}$ for PL SSFO second exposure; $P<0.05$ ) (Figure $6 \mathrm{C}$ ). We reasoned that we should be able to rescue the ability of IL VIPergic neurons to reduce food intake if animals were subsequently given a novel palatable diet that was different in nutrient composition and texture (HCD2) from the initial diet (HCD1) used in testing. As predicted, IL VIPergic stimulation produced a reduction in palatable food intake following the first but not the second exposure to HCD2 (2-way repeated measures ANOVA, $F_{1,11}=6.8, P=0.0244$. First HCD2 exposure: $2.34 \pm 0.19 \mathrm{kcal}$ for IL SSFO control, $1.73 \pm 0.12 \mathrm{kcal}$ for IL SSFO, $P<0.05$. Second HCD2 exposure: $2.46 \pm$ $0.1 \mathrm{kcal}$ for IL SSFO control, $2 \pm 0.2 \mathrm{kcal}$ for IL SSFO, $P=0.125$ ) (Figure 6B). PL VIPergic stimulation produced a reduction in palatable food intake during the first exposure, an effect that was also maintained during the second exposure (2-way repeated measures ANOVA, $F_{1,12}=20.11, P=0.0007$. First HCD2 exposure: $4 \pm 0.24$ $\mathrm{kcal}$ for PL SSFO control, $3.05 \pm 0.29 \mathrm{kcal}$ for PL SSFO, $P<0.05$. Second HCD2 exposure: $3.56 \pm 0.2 \mathrm{kcal}$ for PL SSFO control, $2.52 \pm 0.33 \mathrm{kcal}$ for PL SSFO, $P<0.05$ ) (Figure 6D).

Although these experiments demonstrate the role of food valuation in driving food intake, our studies using HCD1 and HCD2 also demonstrate how the initial stimulation paradigm and food intake experiment conducted with HCD1 did not simply produce an irreversible change in neuron function that blunted the ability of IL VIPergic stimulation to affect food intake during the second exposure to HCD1. Furthermore, our experiments examining chow food intake in fasted animals suggests that the effect of VIPergic neuron stimulation was not dependent upon disruption of sensory processing; our stimulation affected chow intake only when the perceived value of this food was high. 


\section{PL SSFO Stimulation}
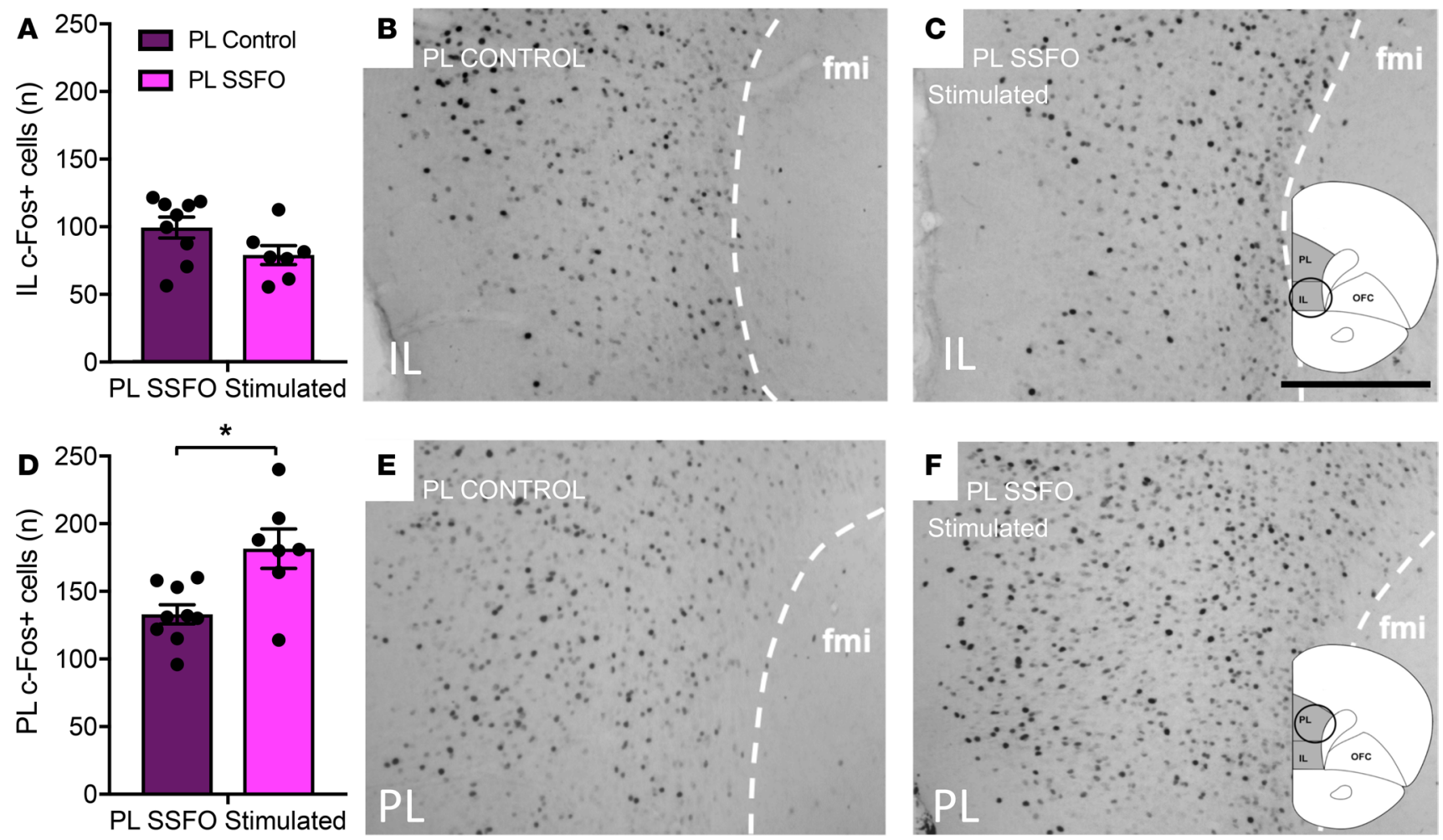

Figure 3. SSFO stimulation of PL VIPergic neurons produces a selective increase in c-Fos expression throughout the PL. PL SSFO stimulation results in an increase in c-Fos expression (F) when compared with controls (E) quantified in (D). PL SSFO stimulation does not result in an increase in c-Fos expression in the IL, when stimulated animals (C) are compared with controls (B) quantified in (A). Scale bar: $200 \mu \mathrm{m}$. Fmi, forceps minor corpus callosum. ${ }^{*} P<$ 0.055, unpaired $t$ test. $10 \mathrm{PL}$ control and 7 PL SSFO mice (A and $\mathbf{D})$ were used for analysis.

We next investigated whether our observations could be extended beyond the 30 -minute testing period and conducted a chronic palatable food intake experiment (using HCD2) that lasted for 24 hours. Based on our acute food intake data, we hypothesized that chronic PL VIPergic stimulation would produce a substantial, prolonged reduction in food intake, while IL VIPergic stimulation would be expected to produce minimal effect because animals had been previously exposed to the diet. Indeed, cumulative 24-hour consumption of HCD2 was significantly reduced following continuous PL but not IL VIPergic stimulation, measured at 30-minute intervals (13.9 $\pm 0.75 \mathrm{kcal}$ for PL SSFO controls, $10.81 \pm 1 \mathrm{kcal}$ for PL SSFO, $P<0.05$, Figure $6 \mathrm{G}$ ). Interestingly, we determined that the rate of food intake over the 24-hour period was significantly different between SSFO- and control-treated animals following either IL VIPergic stimulation (CT0-12, average difference $0.482 \mathrm{kcal} / \mathrm{h}$ with $P=0.0112$; CT13-24, average difference $0.755 \mathrm{kcal} / \mathrm{h}$ with $P=0.0308$, Figure $6 \mathrm{E}$ ) or PL VIPergic stimulation (CT0-12, average difference $1.048 \mathrm{kcal} / \mathrm{h}$ with $P=5.74 \mathrm{e}-09 ; \mathrm{CT} 13-24$ average difference $2.527 \mathrm{kcal} / \mathrm{h}$ with $P=5.12 \mathrm{e}-14$, Figure $6 \mathrm{~F}$ ). However, because only PL stimulation produced a statistically significant reduction in cumulative 24-hour food consumption, this suggests that chronic IL VIP stimulation could produce a small change in food intake that might be measurable at some time point beyond our 24-hour test period. Summarily, these data suggest that IL and PL VIP neurons affect palatable food intake through behaviorally separate pathways.

Activation of IL but not PL VIP neurons suppresses interest in other natural novel stimuli not associated with food intake. The selective effect of IL VIPergic neuron stimulation on the intake of a highly valued, novel food suggested that IL VIPergic neurons might also control novel social- and novel object-driven investigatory behavior. Thus, we tested whether interaction with a novel object or novel mouse was altered following VIPergic activation. In agreement with our observations on food intake, mice that underwent VIPergic stimulation in the IL exhibited decreased time spent investigating a novel object in an open field 

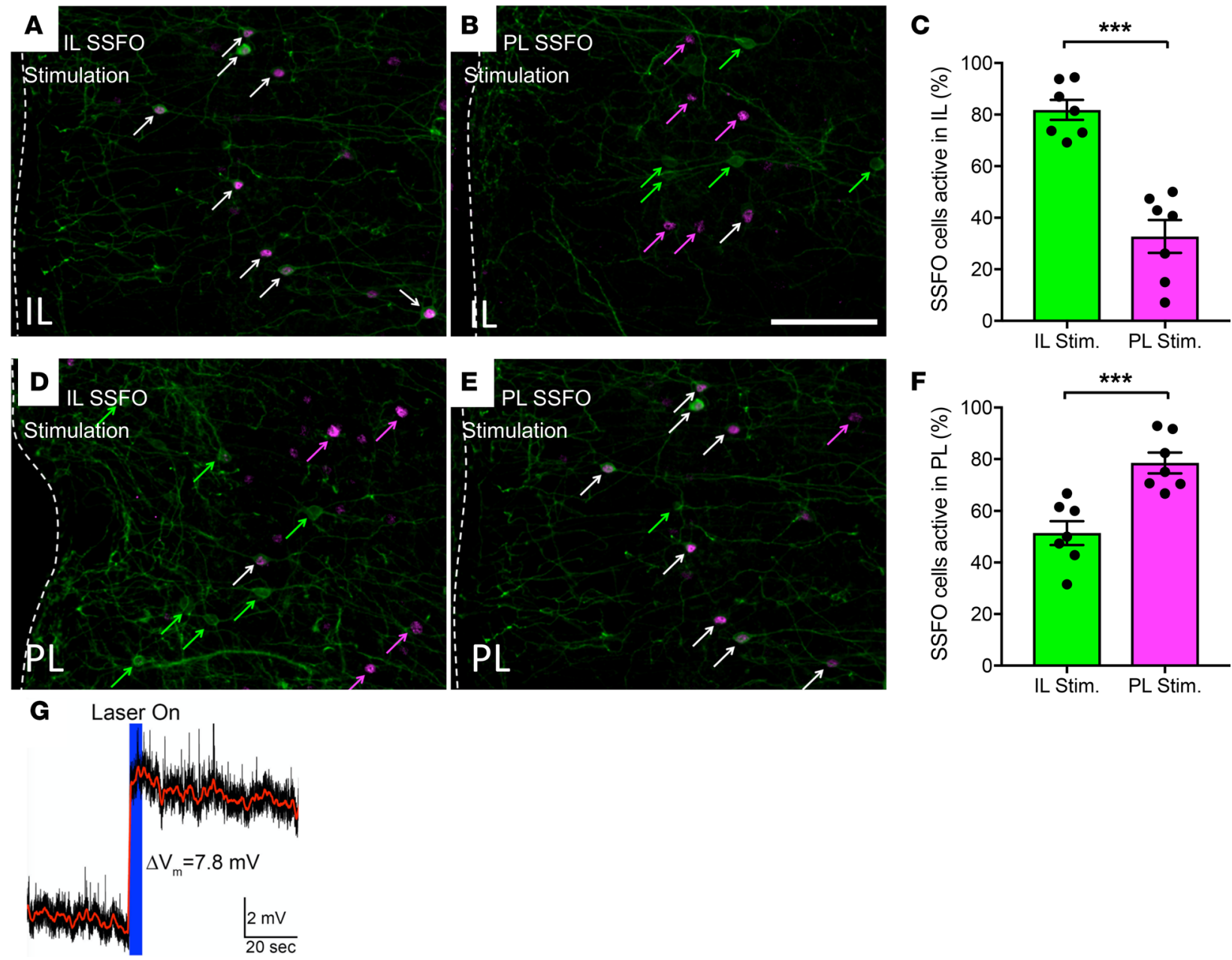

Figure 4. Selective stimulation of IL and PL VIPergic neurons of the mPFC. Activation of an SSFO-YFP driven by 473-nm laser in the IL significantly enhances c-Fos expression in VIPergic neurons of the IL (A) when compared with when SSFO stimulation occurs in the PL (B) as quantified in (C). Similarly, activation of the SSFO in the PL significantly increases VIPergic c-Fos expression in the PL (E) compared with when SSFO stimulation occurs in the IL (D) as quantified in (F). Green arrows denote YFP+ cells, magenta arrows denote c-Fos ${ }^{+}$cells, and white arrows denote double-labeled cells. Scale bar: $200 \mu \mathrm{m}$. (G) Representative trace showing SSFO-driven VIP neuronal depolarization. ${ }^{* *} P<0.0015$, by unpaired $t$ test. 7 IL SSFO and 7 PL SSFO mice (A and $\left.\mathbf{D}\right)$ were used for analysis.

with respect to nonstimulated control counterparts. For IL SSFO controls: $115.1 \pm 13.7$ seconds spent in center with no object, $207.7 \pm 21.9$ seconds spent in center with novel object present, $P<0.001$. For IL SSFO: $150 \pm 16$ seconds spent in center with no object, $158.5 \pm 32.8$ seconds spent in center with novel object present, $P=0.82$ (Figure 7A). PL VIPergic stimulation had no effect on novel object investigation. For PL SSFO controls: $115.7 \pm 10.7$ seconds spent in center with no object, $221 \pm 25.7$ seconds spent in center with novel object present, $P<0.01$. For PL SSFO: $126.7 \pm 13.3$ seconds spent in center with no object, $230.8 \pm 51.4$ seconds time in center with novel object present, $P<0.05$ (Figure $7 \mathrm{~B}$ ). Similarly, IL VIPergic stimulation decreased interest in investigating a novel mouse in an open field (43.46 \pm 6.6 seconds for IL SSFO control, $8.35 \pm 4$ seconds for IL SSFO, $P<0.001$, Figure 7C) while PL VIPergic stimulation was not associated with any changes in social interaction $(63.98 \pm 6.09$ seconds for PL SSFO control, $50.51 \pm 11.41$ seconds for PL SSFO, Figure 7C). Taken together, these results suggest that IL VIP stimulation may act to decrease the salience or value of both novel food and other natural stimuli.

Neither IL nor PL VIP activation induces anxiety-like behavior. Although we observed that mPFC VIPergic activation reduced the value of food and other rewarding stimuli, it is also possible that the observed effects may have resulted from a confounding change in other behaviors that the frontal cortex regulates. Although the lack of effect of either VIPergic IL or PL manipulation on motivation (Figure 5D) suggests that no increase in a depression-like or high-stress state occurred (31-33), it is possible, based on prior work conducted in the rodent $\operatorname{mPFC}(34,35)$, that anxiety-like behavior may have been increased following our stimulation paradigm. To test 
A

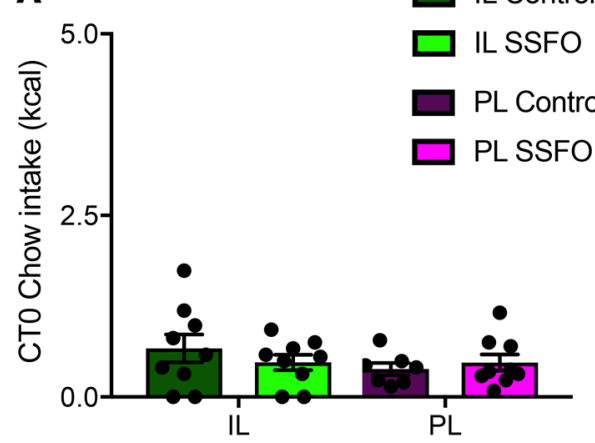

D

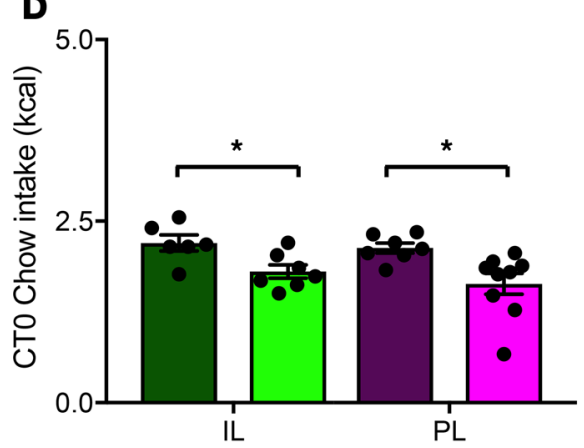

$\mathbf{F}$

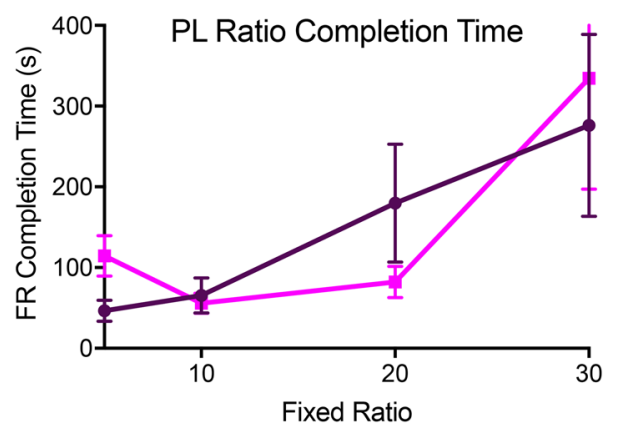

B

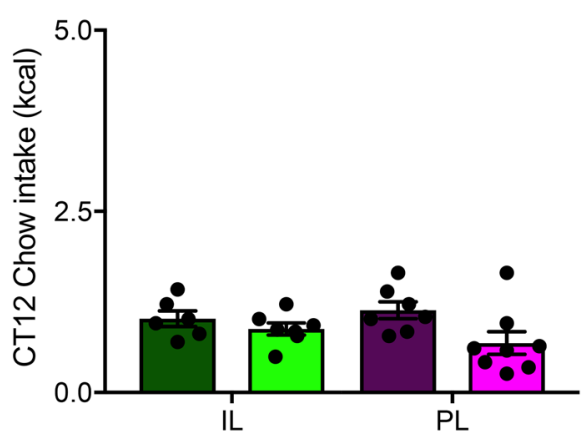

E

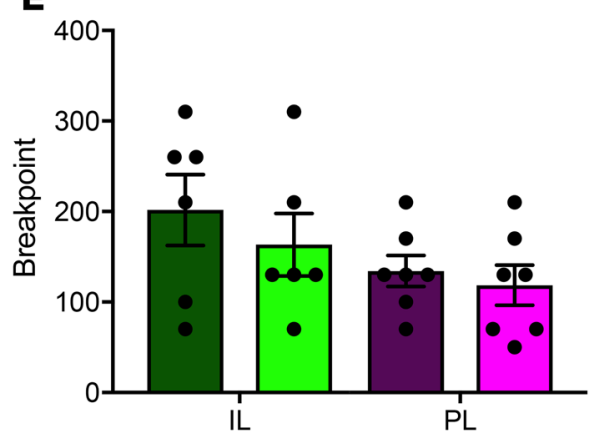

G

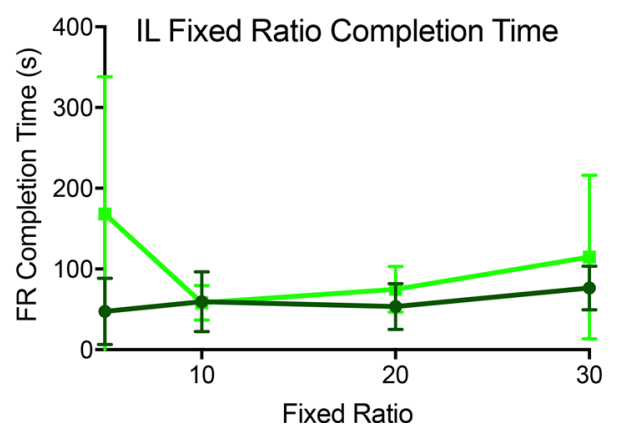

Figure 5. VIPergic stimulation in the IL and PL reduces HCD intake while having no effect on low-calorie rodent chow consumption or on the motivation to obtain food reward. Intake of low-calorie rodent chow at either CTO (A) or CT12 (B) was unaffected by either IL or PL VIPergic stimulation. Both IL and PL VIPergic stimulation reduced the intake of HCD1 over a period of 30 minutes at CTO when compared with control-stimulated mice (C). Similarly, both IL and PL VIP stimulation reduced chow intake following an 18-hour fast (D). Progressive ratio responding for chocolate-flavored pellets was unchanged following either IL or PL VIPergic stimulation, as measured by ratio breakpoint (E) or as time to ratio completion (F and $\mathbf{G})$. ${ }^{*} P<0.05$; ${ }^{* *} P<0.015$, by 2 -way repeated-measures ANOVA with Tukey's post hoc comparison, with treatment and time points as independent variables. 6-9 IL control, 6-9 IL SSFO, 7 PL control, and 7-9 PL SSFO mice were used for analysis.

this possibility, mice expressing SSFO in either IL or PL VIPergic neurons were both subjected to an open field assay and tested using an elevated plus maze to elucidate changes in anxiety-like behavior. No effect of either stimulation on time spent in the center of an open field (Figure 8A) or time spent in open arms of an elevated plus maze (Figure $8 \mathrm{C}$ ) was observed, suggesting that $\mathrm{mPFC}$ VIP stimulation does not alter anxiety behavior. Interestingly, both IL and PL VIP stimulation decreased total distance traveled in an open field (5430 $\pm 431 \mathrm{~cm}$ for IL SSFO controls, $4208 \pm 336 \mathrm{~cm}$ for IL SSFO, $P<0.05 ; 5393 \pm 300 \mathrm{~cm}$ for PL SSFO controls, $4087 \pm 509$ $\mathrm{cm}$ for PL SSFO, $P<0.05$, Figure $8 \mathrm{~B}$ ), a result that demonstrates the complexity of VIPergic regulation of IL and PL function. Although VIP neurons act through different mechanisms to reduce food intake in the IL and $\mathrm{PL}$, they appear to act in both areas of the $\mathrm{mPFC}$ to reduce exploratory behavior in a novel environment.

IL and PL VIPergic activation produces distinct changes in subcortical neuronal activation. Because our data indicate that VIP neurons of the IL and PL can produce a reduction in palatable food intake that is differentially sensitive to the novelty of the food source, we examined how VIP stimulation altered neuron 

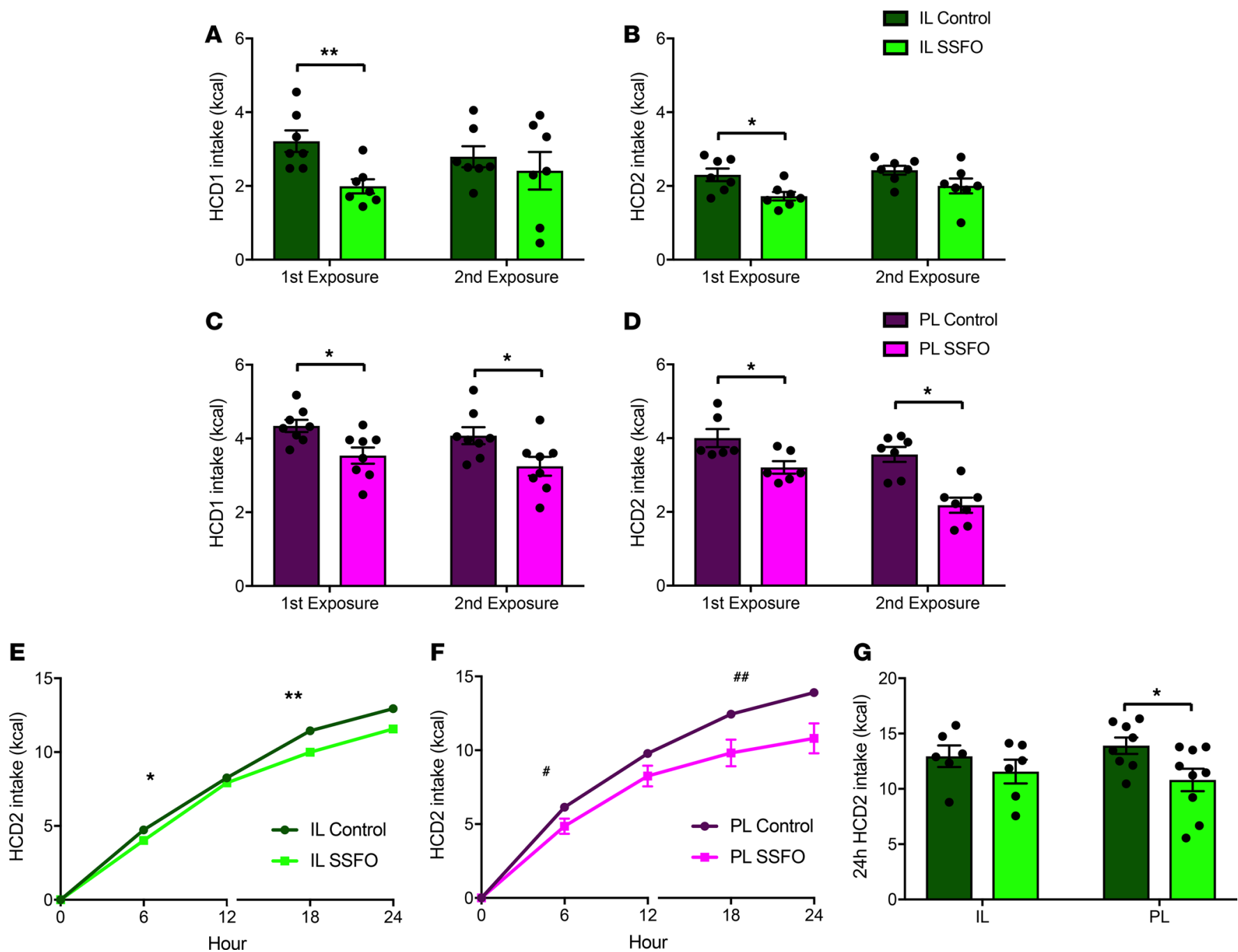

Figure 6. IL but not PL VIPergic stimulation-driven reduction in HCD intake is dependent upon food novelty while showing little effect on 24-hour food intake. Although stimulation of IL VIPergic neurons produced a reduction in food intake of HCD1 during initial testing, these neurons produced no change in feeding when retested using the same diet (A). The effect of IL VIPergic stimulation to reduce food intake can be rescued, however, when animals are subsequently tested using a novel HCD (HCD2) (B, left). Again, when animals were retested using this same diet, IL VIPergic stimulation had no effect on food intake (B, right). PL VIPergic stimulation produced a reduction in HCD intake regardless of whether the diet was novel, when tested using either HCD1 (C) or HCD2 (D). IL VIPergic stimulation for 24 hours exerted a minimal effect on food intake (E and $\mathbf{G}$, left) while PL VIPergic stimulation produced a significant reduction in consumption ( $\mathbf{F}$ and $\mathbf{G}$, right). ${ }^{*} P<0.05$; ${ }^{* *} P<0.01$; ${ }^{*} P<10^{-8}$; ${ }^{\# \#} P<10^{-13}$ by 2-way repeated-measures ANOVA with Tukey's post hoc comparison, with treatment and time points as independent variables (A-D), linear regression models were created with time and treatment group as fixed effects (separate models were created for light hours [0-12] and dark hours [13-24]) (E and F), or unpaired 2-tailed $t$ test (G). 7 IL control, 7 IL SSFO, 8 PL control, and 7 PL SSFO mice were available for analysis.

activation in subcortical brain areas, hypothesizing that the c-Fos expression patterns from IL or PL VIP-stimulated neurons would differ significantly. Indeed, only the dorsomedial hypothalamic nucleus (DM) was shown to exhibit a change in c-Fos expression that was similar between the IL and PL stimulation paradigms; both stimulations significantly increased c-Fos expression in this area (Table $1, P<$ 0.01 for IL; $P<0.05$ for PL). Although IL VIPergic activation significantly increased c-Fos expression in the core of the AcbC and the CeM, no changes in c-Fos expression in these brain structures were observed following PL VIPergic activation. Interestingly, PL activation produced a significant increase in the expression of c-Fos in the PVN while decreasing c-Fos expression in the ARC. Again, IL VIPergic stimulation did not change c-Fos expression in either of these structures. These data demonstrate how manipulations of IL and PL VIPergic neurons produce distinctive changes in subcortical neuronal activation that likely explain observed differences in behavior. 

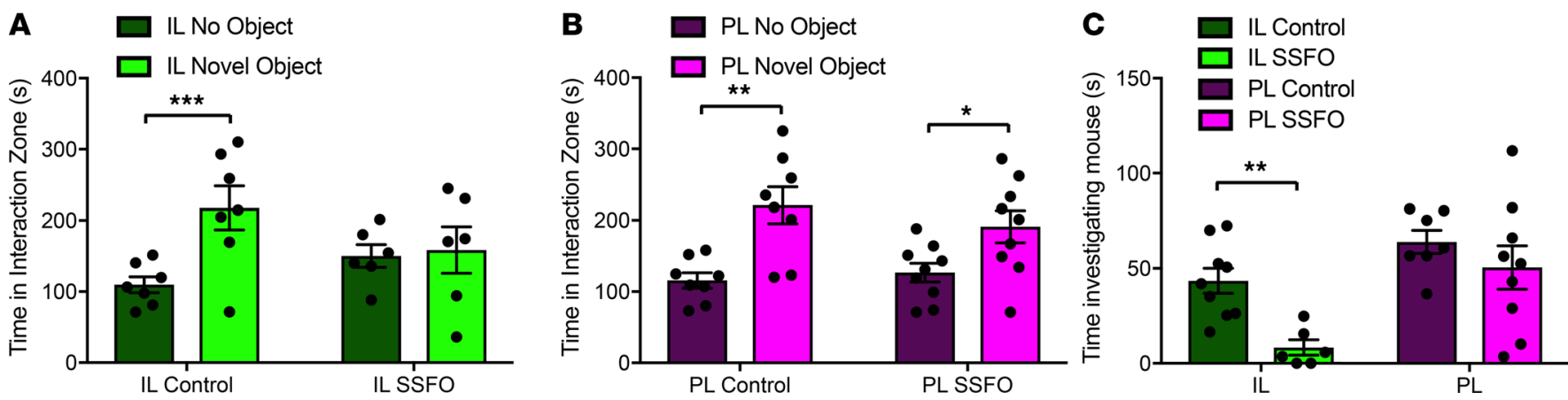

Figure 7. IL but not PL VIPergic stimulation reduces novel object and novel social investigatory behavior. SSFO-driven stimulation of IL VIPergic neurons reduced novel object investigation when compared with controls (A). PL VIPergic stimulation had no effect on novel object investigatory behavior (B). IL VIPergic stimulation reduced time spent investigating a novel conspecific animal while PL VIPergic stimulation exhibited no effect (C). ${ }^{*} P<0.05 ;{ }^{* *} P<$ $0.01 ;{ }^{* *} P<0.0015$ by unpaired 2-tailed $t$ test. 9 IL control, 6 IL SSFO, 8 PL control, and 9 PL SSFO mice were available for analysis.

\section{Discussion}

Our data represent the first description to our knowledge of an interneuronal cell type in the cortex that is capable of regulating food intake. To our knowledge, this is also the first description of a cellular pathway capable of selectively modulating palatable food intake while having no effect on standard chow consumption in sated animals. Although prior published reports have described the role of the frontal cortex in controlling feeding, no studies to our knowledge in humans or in animal models have shown how activation of a defined cortical interneuron cell type could alter ingestive behavior. Our studies provide an explanation at the cellular level of how aspects of reward behavior can be regulated by a defined neuronal cell type; mPFC VIPergic neuron stimulation can selectively reduce the intake of a highly valued food while having no effect on the motivation to work for food reward.

Prior studies have shown how VIPergic neuronal activity in both the frontal and auditory cortices is elevated following receipt of either reward or punishment $(7,22)$; these neurons respond following action outcome, suggesting that they act to relay a feedback signal. In this way, VIPergic activation would be expected to modulate the positive reward prediction signal associated with the presentation of a novel food or other stimulus, updating the perceived value of the stimulus. Furthermore, this action as a reward prediction error signal would not be expected to affect motivation because any such signal would likely become attenuated over multiple cycles of reward consumption. Indeed, our data partly agree with this hypothesis: VIP neurons in the IL likely regulate high-value food intake through mediating the strength of a reward prediction signal error that would likely result from the consumption of a novel food or a food that undergoes an alteration in perceived value, while having no effect on the motivation to obtain food reward. In the absence of the generation of this hypothetical reward prediction error signal, for example when the same diet is presented multiple times, VIP depolarization in the IL would be expected to exert little effect on feeding.

This interpretation of the function of IL VIP neurons is also in agreement with our observation that these cells, when activated, can also reduce the intake of chow in a fasted animal. In this case, the effect of VIP neuron stimulation can be seen only when chow value is increased through fasting, potentially altering the predicted value of this food, while in the fed state, IL VIP stimulation has no effect on food intake. It has yet to be determined, however, whether IL VIP neuron stimulation amplifies a hypothetical error prediction signal that might result from the elevation in value of chow food that is ingested in the fasted state.

Meanwhile, the reduced palatable food intake we examined after PL VIPergic activation (Figure $5 \mathrm{C}$ and Figure 6, C and D) is consistent with the action of these neurons to reduce food intake through modulation of the salience of, value of, or attention to the food source. Although further studies are required to determine which of these processes engaged by reward presentation are altered by changes in PL VIPergic neuron activity, we can conclude from our data that PL VIPergic reduction in palatable food intake is not novelty dependent. This suggests that our stimulation in the PL does not modulate a reward prediction error signal. Interestingly, although the novelty value of palatable food does not appear to be the principal driver of a reduction in intake by the PL VIP neurons, these cells can act to reduce explor- 

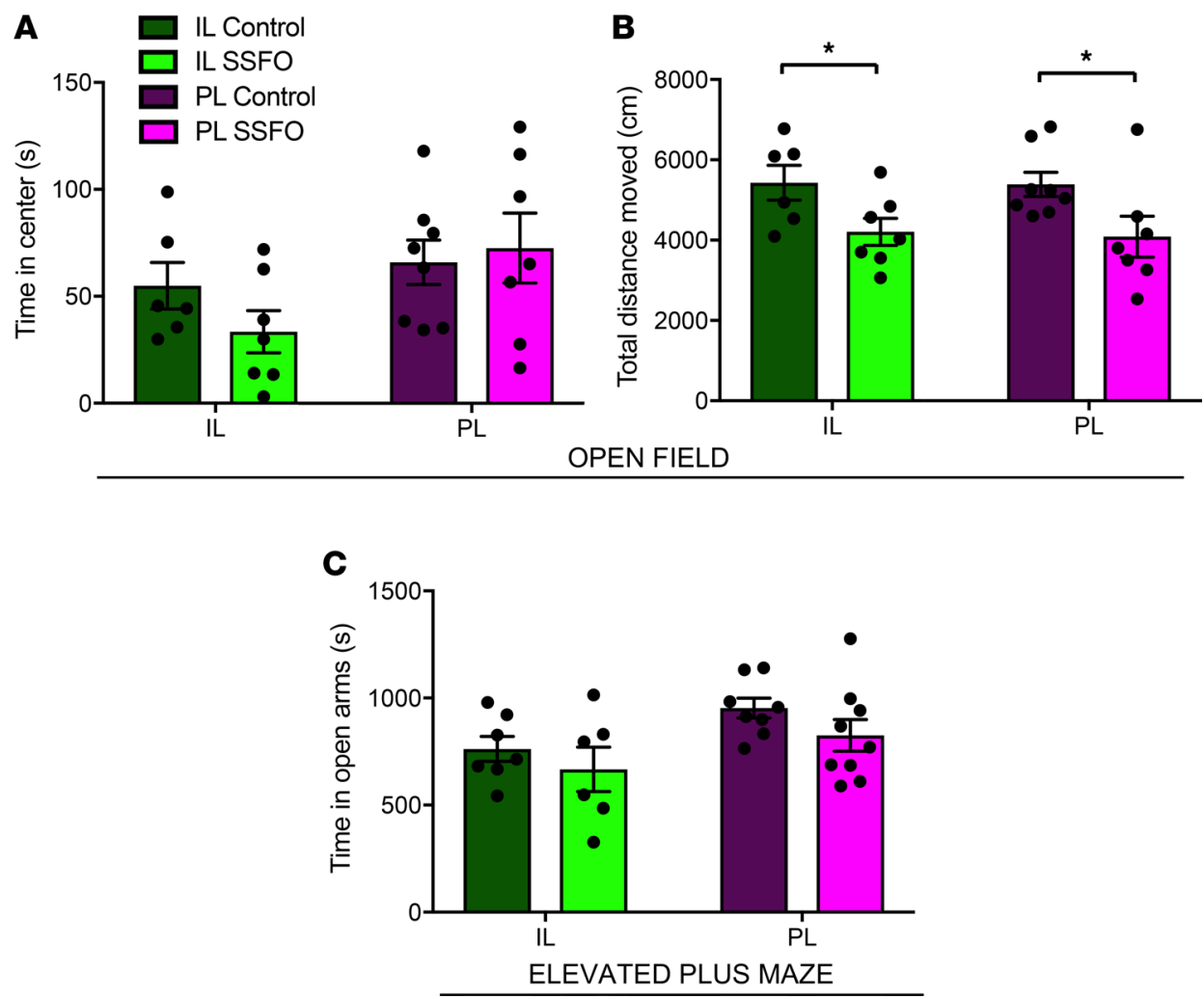

Figure 8. Neither IL nor PL VIPergic stimulation affects the expression of anxiety-like behavior. No effect of IL or PL VIPergic stimulation was seen on time spent in the center of an open field arena (A) or in time spent in the open arms of an elevated plus maze (C). However, both IL and PL VIPergic activation reduced novel environment-driven exploratory behavior in the open field arena (B). ${ }^{*} P<0.05$ by unpaired 2 -tailed $t$ test. 6 IL control, 7 IL SSFO, 8 PL control, and 9 PL SSFO mice were available for analysis.

atory locomotion in a novel environment, as we have also observed with IL VIPergic stimulation. Thus, our data demonstrate how mPFC VIP cells can regulate the response to select novel stimuli, an effect that is likely dependent upon the behavioral selectivity of the IL and PL brain regions.

Our c-Fos expression data provide an explanation of how manipulations of IL and PL VIPergic neurons produce differential effects on food intake and novelty-driven behaviors. PL VIPergic stimulation was shown to reduce c-Fos expression in the ARC, while PVN c-Fos expression was increased, with both areas having been implicated in the control of food intake (36-38). Although direct activation of these target sites is unlikely, an indirect projection such as that from the PFC to the bed nucleus of the stria terminalis (BNST) (39) and subsequently from the BNST to the PVN could mediate the observed increase in c-Fos expression. IL VIPergic activation resulted in significant increases in c-Fos expression in the CeM and AcbC, areas that have been implicated in the regulation of reward valuation and novelty-driven behavior (40-42), in addition to food intake $(11,12,43)$. Although a systematic investigation of PL and IL projections has not been attempted in the mouse, prior work in rats has shown how these brain regions differentially innervate, either directly or indirectly, subcortical structures that include the amygdala and nucleus accumbens $(44,45)$, observations that could explain our described differences in c-Fos expression and animal behavior. The activation of the DM following stimulation of either IL or PL VIPergic neurons might also contribute significantly to the control of food intake because prior studies have shown how activation of select DM neurons can regulate autonomic outflow (46) and food intake (47) in addition to affecting reward-seeking behavior (48). Neither IL nor PL stimulation produced a change in LH c-Fos levels, which was unexpected given the described importance of mPFC projections to the LH in the control of feeding behavior (12). This result may be due to a lack of activation of this projection or may instead be due to a technical issue. Because the mPFC VIPergic activation may be acting to suppress LH activity (12), this effect would be difficult to observe in neurons that, under resting conditions, are quiescent. 
Table 1. Distinct patterns of c-Fos expression are observed throughout the brain following SSFO-driven stimulation of IL and PL VIPergic neurons

\begin{tabular}{lcccccccc}
\hline & \multicolumn{2}{c}{ Contralateral IL } & \multicolumn{2}{c}{ Ipsilateral IL } & \multicolumn{2}{c}{ Contralateral PL } & \multirow{2}{*}{ Ipsilateral PL } \\
& SSFO & Control & SSFO & Control & SSFO & Control & SSFO & Control \\
PVN & $161 \pm 41$ & $218.2 \pm 29$ & $162 \pm 40$ & $204 \pm 26$ & $127 \pm 16$ & $216 \pm 20^{\mathrm{A}}$ & $152 \pm 25$ & $210 \pm 23$ \\
DM & $52 \pm 10$ & $71.2 \pm 4$ & $44 \pm 5$ & $74.2 \pm 7^{\mathrm{B}}$ & $55 \pm 8$ & $73.6 \pm 9$ & $50.5 \pm 5$ & $73.8 \pm 10^{\mathrm{B}}$ \\
Rostral LH & $127 \pm 28$ & $116 \pm 14$ & $129 \pm 15$ & $117 \pm 15$ & $112 \pm 18$ & $99 \pm 17$ & $112 \pm 12$ & $100 \pm 24$ \\
ARC & $48 \pm 10$ & $53 \pm 15$ & $62 \pm 9$ & $56 \pm 7$ & $51 \pm 4$ & $40 \pm 4$ & $60 \pm 3$ & $47 \pm 5^{\mathrm{B}}$ \\
BLA & $37 \pm 3$ & $44 \pm 9$ & $27 \pm 4$ & $37 \pm 6$ & $36 \pm 8$ & $26 \pm 5$ & $36 \pm 6$ & $37 \pm 8$ \\
CeM & $80 \pm 13$ & $107 \pm 25$ & $52 \pm 7$ & $112 \pm 13^{\mathrm{A}}$ & $88 \pm 11$ & $106 \pm 20$ & $96 \pm 20$ & $130 \pm 23$ \\
VTA & $49 \pm 14$ & $35 \pm 7$ & $52 \pm 11$ & $31 \pm 9$ & $34 \pm 6$ & $39 \pm 7$ & $37 \pm 7$ & $39 \pm 3$ \\
AcbC & $237 \pm 40$ & $353 \pm 57$ & $167 \pm 32$ & $314 \pm 41^{\mathrm{B}}$ & $223 \pm 19$ & $230 \pm 41$ & $212 \pm 18$ & $296 \pm 37$
\end{tabular}

IL VIPergic stimulation enhanced c-Fos expression in the dorsomedial hypothalamus (DM), the central nucleus of the amygdala (CeM), and nucleus accumbens core ( $A c b c)$. PL VIPergic stimulation enhanced c-Fos expression in the paraventricular hypothalamic nucleus (PVN) and in the DM, while reducing c-Fos expression in the arcuate nucleus of the hypothalamus (ARC). ${ }^{A} P<0.01$, unpaired 2-tailed $t$ test; ${ }^{B} P<0.05$, unpaired 2-tailed $t$ test. Neither treatment affected c-Fos expression in the lateral hypothalamus (LH), basolateral amygdala (BLA), or ventral tegmental area (VTA).

Finally, although our studies have focused on describing the role of VIPergic neurons in the control of food intake, it is likely that the activity of other interneuronal cell types can also influence aspects of reward valuation or motivation to obtain food. Because one of the main targets of the VIPergic neurons are other GABAergic interneurons that coexpress somatostatin (SST) (49, 50), it is quite likely that activation of this cell type would produce an opposite effect on feeding behavior, enhancing intake. However, because VIPergic neurons also innervate pyramidal neurons directly (51), producing a complex effect on circuit activity, SST activation may not produce a reciprocal change in behavior. Indeed, our observations regarding VIPergic function contrast with those data describing the role of another VIPergic neuronal target, the parvalbumin-expressing (PV) interneurons, suggesting that some of the behavioral consequences of VIPergic activation may be unique to this cell type. Although effects on food intake have yet to be investigated, activation of PV interneurons in the PFC enhances attentional performance (52) while manipulation of this cell type in isolation shows no effect on social interaction (23). These differences between the actions of PV neurons of the frontal cortex and our reported data concerning mPFC VIPergic neurons suggest that our manipulations have revealed an unappreciated behavioral selectivity of this cell type. This selectivity also suggests that our manipulation is not producing a nonspecific effect on network activity but instead produces a specific, behaviorally relevant effect on pyramidal cell output.

When considering potential caveats within the interpretation of our data, it is possible that our manipulation of VIP neuron activity producing a change in behavior did so through a synergy with isoflurane, which was administered in all animals before laser coupling to the ferrule. Although we were careful to begin behavioral testing only 5 minutes following recovery from and washout of isoflurane, it is possible that this induction of anesthesia may have affected behavior. It is also possible that other environmental effects in addition to isoflurane affected the outcome of our feeding experiments in particular, because our control populations appeared to show differences in consumption when tested sequentially. When we subsequently tested another group of PL and IL controls concurrently, however, we were unable to recapitulate the differences in food intake seen between the 2 control populations reported in manuscript (data not shown). Thus, it is quite likely that the observed differences in food intake between PL and IL control groups may have been due to environmental differences present upon testing and likely not due to the surgical manipulation or other aspects of the paradigm.

In conclusion, our studies have identified a potentially unique role for IL and PL VIPergic neurons in the control of palatable food intake. Our work is the first, we believe, to describe the ability of a cortical neuron subtype to selectively reduce intake of highly valued food in the absence of an effect on nonfasted chow intake. In addition, these data demonstrate how novelty-dependent and -independent mechanisms are likely involved in the frontocortical control of feeding behavior. 


\section{Methods}

Experimental animals. We purchased 12-week-old adult male VIP-IRES-Cre-transgenic mice (stock 010908) from The Jackson Laboratory and housed them in the Pinn Hall vivarium at the University of Virginia on a 12-hour light/12-hour dark cycle (lights off at 2100 hours) with ad libitum access to food and standard chow (Teklad 2013, 4\% fat, 17\% protein, 48\% carbohydrate, no sucrose, $2.9 \mathrm{kcal} / \mathrm{g}$ ) unless otherwise noted. The Cre-expressing line was backcrossed to C57BL/6J animals (Jackson Laboratory) for at least 7 generations. Heterozygous males were used for all described experiments and were generated by crossing VIP Cre-expressing males to C57BL/6J females. Animals were genotyped using the following primer pairs: mutant forward: 5'-CCCCCTGAACCTGAAACATA-3'; common: 5'-GGACACAGTAAGGGCACACA-3'; WT forward: 5'-TCCTTGGAACATTCCTCAGC-3'. We used 6-9 mice per treatment group for each experiment that we conducted.

Adeno-associated viral vector and stereotaxic viral injections. VIP neurons were stimulated using a Cre-dependent SSFO virus, AAV5-EF1a-DIO-ChR2 (C128S/D156A)-EYFP (23), sourced from the University of North Carolina Gene Therapy Core Facility. We injected $100 \mathrm{nl}$ of virus bilaterally into either the PL or IL of 8-week-old male VIP-Cre mice using coordinates based on Frankin and Paxinos (53, 54) (PL: $+1.8 \mathrm{~mm}$ from bregma, $\pm 0.4 \mathrm{~mm}$ lateral of midline, and $1.4 \mathrm{~mm}$ ventral of the dura; IL: $+1.8 \mathrm{~mm}$ from bregma, \pm 0.4 $\mathrm{mm}$ lateral of midline, and $1.8 \mathrm{~mm}$ ventral of the dura). We next installed a fiber optic ferrule (FT200EMT, Thor Labs) at the dorsal boundary of the PL (+1.3 mm ventral of the dura) or IL (+1.7 mm ventral of the dura) following viral injection. The ferrule was attached to the skull and held in place using dental adhesive. Animals were allotted 6 weeks to recover from surgery before behavioral experiments were conducted.

SSFO stimulation protocol. Before each experiment, mice were briefly anesthetized with isoflurane, then fiber optically coupled to a diode-pumped, 473-nm blue laser (CrystaLaser model BC-473-060-M) controlled by a function generator (Grass Instruments), which delivered one 5 -second train of $20-\mathrm{Hz}$ pulses, 5 -ms pulse width, with power output of $10 \mathrm{~mW}$. Following stimulation, the laser was uncoupled, and after a period of 5 minutes to recover from handling, mice underwent all experimentation in the absence of tethering. To control for stress associated with handling, mice were acclimated to this stimulation procedure in the absence of actual stimulation once daily for 1 week before any experimentation. Control animals were subjected to this tethering procedure for each experiment, minus laser stimulation.

Free feeding assays. All behavioral testing for palatable chow and standard chow feeding assays occurred in the home cage in the home room, except the 24-hour feeding assay (described below). For the 30-minute free feeding on standard chow, mice had their food removed at lights on and were tethered to the laser as described above; then laser stimulation was either applied or withheld depending on treatment condition. After 5 minutes, mice received a novel piece of preweighed standard chow, and food intake was quantified at 30 minutes, after which food was removed and mice resumed ad lib chow feeding. For feeding during the dark cycle, procedures were identical, except the experiment began at CT12 as opposed to CT0.

Measurement of palatable food intake was performed as previously described (55). On the night before testing, mice received a small $(<0.1 \mathrm{~g}$ ) sample of the HCD of interest (HCD1: Teklad TD.88137, 21\% fat, $17.3 \%$ protein, $48.5 \%$ carbohydrates, $4.5 \mathrm{kcal} / \mathrm{g}$; HCD2: Research Diets Inc., D12331, 58\% fat, 17\% protein, $25 \%$ carbohydrates, $5.56 \mathrm{kcal} / \mathrm{g}$ ) to reduce aversion. At CT0 all food was removed, and mice were tethered to the laser; then laser stimulation was either applied or withheld depending on treatment condition. After 5 minutes, mice were challenged with approximately $3 \mathrm{~g}$ of preweighed HCD and allowed to consume. After 30 minutes, food was removed and weighed; then mice were returned to ad lib chow feeding. Mice were assigned randomly to the treatment or control conditions. Mice were given 4 days to recover from the binge before subsequent HCD exposure. For mice to serve as their own controls when comparing HCD consumption across multiple exposures, animal treatments remained consistent across days.

Measurement of 24-hour food intake. Mice were stimulated with one 5-second train of 20-Hz pulses, 5-ms pulse width, with power output of $10 \mathrm{~mW}$, once every 30 minutes throughout the 23-hour duration of this study. To minimize disturbing the mice during stimulation, mice remained tethered to the laser via a fiber optic cable coupled to an optical commutator for the duration of the study. Mice were placed in cages modified for this setup in a separate room used for behavioral studies for this study, and they were acclimated to the cages 24 hours before testing. At lights on, mice were tethered to commutators, then given free access to the HFD of interest after 1 hour. Laser stimulation occurred at the onset of diet presentation, then every subsequent 30 minutes for 23 hours. Food intake was quantified every hour, and body weight was quantified every 6 hours; mice remained tethered during body weight quantification. 
Operant responding for palatable food reward. Behavioral testing for the operant conditioning assay occurred in a dedicated behavior room, separate from the home room, conducted as we described previously (10). Unlike testing during the other described behavioral experiments, mice remained tethered for the duration of the training sessions and the experiment, receiving laser stimulation during the latter at 15-minute intervals. Testing was performed in sound-attenuated boxes (Med-Associates). Each box was equipped with 3 nose poke holes arranged in a line on one side of the chamber and a food magazine on the opposite side. Each nose poke hole, as well as the magazine, was equipped with an infrared beam break detector. Three days before training, mice were placed on food restriction, with access to regular chow for $3 \mathrm{~h} /$ day, such that they were maintained at $80 \%$ original body weight. Mice were trained for $1 \mathrm{~h} /$ day, every day. Before any training, mice initially underwent extinction training to extinguish any innate preferences for any of the nose poke holes. In this phase, a nose poke did not result in any reward delivery. After passing extinction (fewer than 10 pokes in any hole in a given session), mice were passed on to FR training. FR training proceeded in 3 stages: FR1, FR3, and FR5. In FR1, a single nose poke in the center hole resulted in delivery of a palatable food reward (Bio-Serv, F05301, 5.6\% fat, 18.7\% protein, $59.1 \%$ carbohydrates, $3.6 \mathrm{kcal} / \mathrm{g}$ ) to the magazine. In FR3, 3 nose pokes were required for a reward, and in FR5 5 nose pokes were required. A mouse was considered to have passed a stage when it attained 30 rewards in a single session. Mice were moved from FR1 to FR3 after passing FR1 1 time, moved from FR3 to FR5 after passing FR3 twice, and moved from FR5 to progressive ratio (PR) testing after passing FR5 3 times. In PR testing, the number of nose pokes required for a reward increased progressively during a session, on the following schedule: 5 pokes for the first reward, then 10, 20, 30, 50, 70, 100, 130, $170,210,260,310$, and finally 370. PR testing lasted for a maximum of 2 hours and could terminate early if the mouse did not complete any given stage in 30 minutes or less. Total nose pokes recorded until this point were reported as "breakpoint." Mice underwent 2 days of PR testing on food restriction alternating laser/control treatment, with half receiving the laser stimulation on the first day and half receiving no stimulation, such that each animal underwent PR testing on each treatment. The chambers were cleaned between each trial with Minncare disinfectant to remove residual odors.

Open field. The open field assay was conducted as previously described (10). The lights in the behavioral room were turned down, and mice were allowed to acclimate for at least 1 hour before testing. Five minutes after laser stimulation, mice were placed into the open field chamber and allowed to explore for 15 minutes while movement was recorded using EthoVision XT tracking software (Noldus). The open field chamber was cleaned between each mouse with Minncare disinfectant to remove residual odors. During testing, the lights in the room remained turned down, providing a dim light environment. All mice underwent this experiment before any other experiments in this open field arena so that arena novelty did not exist as a confounding variable during social interaction and novel object assays.

Social interaction. The social interaction task was performed in our open field chamber, as previously described (56). Before the social interaction test, all mice were brought to the behavior room and allowed to acclimate for at least 1 hour. Mice received laser stimulation 5 minutes before testing. In brief, the chamber was prepared with an empty restrainer in the interaction zone, against the wall. The test mouse was placed in the chamber adjacent to the wall opposite the restrainer (as in the open field assay) and allowed to explore for 150 seconds. The test mouse was then removed to the home cage for 30 seconds, while the empty restrainer was replaced with a new, clean restrainer. A novel mouse (129/SJL, Jackson Laboratory) was placed in the new restrainer, and the test mouse was returned to the chamber and allowed to explore for 150 seconds. Behavior and motion were recorded using EthoVision. The chamber was cleaned between each mouse with Minncare disinfectant to remove residual odors.

Novel object interaction. The novel object interaction assay was also performed in our open field chamber, described above. Before the novel object test, all mice were brought to the behavior room and allowed to acclimate for at least 1 hour. Mice received laser stimulation 5 minutes before testing. In brief, following an initial 15 minutes of exposure to the chamber, mice were reintroduced to the chamber that then contained a novel piece of copper piping. Exploration of the piping, placed in the center of the arena, was recorded using EthoVision.

Elevated plus maze. Anxiety-like behavior was measured using an elevated plus maze apparatus according to our prior published procedure (57). The $12 \mathrm{~cm} \times 50 \mathrm{~cm}$ maze was elevated $55 \mathrm{~cm}$ from the floor in a low-light environment. Time in the open arm was measured during a 5-minute period. Scoring was done automatically using EthoVision. 
Brain tissue preparation and immunohistochemistry. Immunohistochemistry was performed as previously described (55). Mice received either laser stimulation or nothing (controls) in the absence of food 90 minutes before sacrifice by transcardial perfusion fixation. After receiving anesthesia, mice were briefly flushed with buffered saline, followed by perfusion with $4 \%$ paraformaldehyde in 0.1 -M phosphate buffer. Brains were dissected and sectioned at $40-\mu \mathrm{m}$ thickness on a vibratome (Leica Biosystems). Immunoperoxidase staining was used to visualize c-Fos expression. All primary and secondary antibody solutions were made in $0.01 \mathrm{M}$ PBS containing $0.5 \%$ Triton $\mathrm{X}-100,1 \%$ normal goat serum, and $0.1 \%$ sodium azide with the following dilutions: rabbit anti-Fos (Ab-5, 1:5000, MilliporeSigma, PC38), goat anti-rabbit IgG (1:1000, Jackson ImmunoResearch Laboratories Inc.). After 48 hours of incubation in primary antibody, sections were washed in PBS and incubated in goat anti-rabbit IgG secondary antibody overnight at $4^{\circ} \mathrm{C}$. Following a subsequent series of washes in PBS, sections were incubated for 3 hours in avidin-biotin-peroxidasa complex diluted in PBS with 0.1\% Triton x-100 (ABC Elite kit, 1:1000, Vector Labs). After a final series of washes, sections were visualized with nickel-enhanced DAB tablets (SIGMAFAST, Sigma-Aldrich) to yield a black color. For fluorescent immunohistochemistry, sections were incubated in a mix of chicken anti-GFP (Aves Labs catalog GFP-1020, 1:2000) and rabbit anti-Fos (Ab-5, 1:5000, MilliporeSigma, catalog PC38) polyclonal antibodies for 24 hours, followed by Alexa Fluor 488-conjugated goat anti-chicken and Cy3-conjugated goat anti-rabbit IgG (Jackson ImmunoResearch Laboratories Inc.; 1:1000) overnight in a light-protected container. Sections from each were then mounted in sequential order, air-dried, dehydrated, cleared, and coverslipped in Vectashield hard-set mounting medium (Vector Laboratories). During the entire process the slides were protected from light exposure.

Quantitative analysis of c-Fos immunoreactivity. In the series stained for only c-Fos, numbers of stained cell nuclei in the mPFC were counted using the ImageJ (NIH). The mPFC area analyzed corresponded closely with the coronal diagram at $1.78 \mathrm{~mm}$ anterior to the bregma (50). Quantitation was done in the NIH's ImageJ, where images were equalized in brightness (background subtraction), the threshold was set for binary conversion, and the number of particles (corresponded to labeled nuclei) was counted and recorded. Similarly, c-Fos expression was assessed in the AcbC (bregma $+1.34 \mathrm{~mm}$ ), PVN and LH (bregma $-0.58 \mathrm{~mm}$ ), BLA and CeM (bregma $-1.22 \mathrm{~mm}$ ), DM and ARC (bregma $-1.58 \mathrm{~mm}$ ), and VTA (bregma $-3.08 \mathrm{~mm}$ ).

Quantitative analysis of fluorescent double-labeled cells. The series of sections fluorescently double-labeled for c-Fos and YFP reporter immunofluorescence was evaluated using Neurolucida 2017 (MBF Bioscience) by capturing pairs of images with $\mathrm{a} \times 20$ objective with the excitation/emission filters alternating between Cy 4 and Alexa 488 fluorophores. Then the sections were quantified for double-labeling manually by a blinded technician.

Electrophysiology. In vitro slice preparation and intracellular recordings were performed as described previously (58) with some modifications. Briefly, VIP-Cre mice were stereotactically injected with AAV5EF1a-DIO-ChR2 (C128S/D156A)-EYFP into the mPFC. Animals were allowed to recover for 2 weeks and were then injected with pentobarbital and transcardially perfused with an ice-cold solution of (in $\mathrm{mM}$ ) $92 \mathrm{NMDG}, 26 \mathrm{NaHCO}_{3}, 25$ glucose, $20 \mathrm{HEPES}, 10 \mathrm{MgSO}_{4}, 5$ sodium ascorbate, 3 sodium pyruvate, 2.5 $\mathrm{KCl}, 2$ thiourea, $1.25 \mathrm{NaH}_{2} \mathrm{PO}_{4}$, and $0.5 \mathrm{CaCl}_{2}$ titrated to $\mathrm{pH}$ 7.4. Coronal sections were obtained using a VT1200 vibratome (Leica Biosystems), incubated in aCSF (in mM: $126 \mathrm{NaCl}, 26 \mathrm{NaHCO}_{3}, 10$ glucose, $2.5 \mathrm{KCl}, 2 \mathrm{CaCl}_{2}, 1.25 \mathrm{NaH}_{2} \mathrm{PO}_{4}$, and $1 \mathrm{MgSO}_{4}$ ), held at room temperature, and equilibrated with $95 \%$ $\mathrm{O}_{2} / 5 \% \mathrm{CO}_{2}$. Intracellular recordings were obtained from tissue slices submerged in a chamber perfused with $31^{\circ} \mathrm{C}$ to $33^{\circ} \mathrm{C}$ aCSF. VIPergic neurons expressing the SSFO were identified first by green fluorescent reporter expression and DIC optics using a Zeiss Axio Examiner.A1 microscope and scientific CMOS camera (ORCA-Flash 4.0, Hamamatsu). Recording pipettes were made from borosilicate pipettes pulled using a Sutter P1000 puller. The internal solution was composed of (in $\mathrm{mM}$ ) $130 \mathrm{KCl}, 1 \mathrm{MgCl}_{2}, 0.07 \mathrm{CaCl}_{2}, 10$ HEPES, and 0.1 EGTA, pH 7.3, osmolarity 300 mOsm. Pipettes used tip resistances of 3-4 M. Neurons were stimulated using a diode-pumped, 473-nm blue laser (CrystaLaser model BC-473-060-M), with light delivered through the camera port, controlled by a function generator (Grass Instruments), which delivered one 5 -second train of $20-\mathrm{Hz}$ pulses, 5 -ms pulse width, with power output of $10 \mathrm{~mW}$. Data were acquired using pClamp software (Molecular Devices) using a Multiclamp 700B amplifier. Data were low-pass filtered at $2 \mathrm{kHz}$ and digitized at $10 \mathrm{kHz}$ using a Digidata 1440A (Molecular Devices). Analysis of the effect of laser stimulation on membrane potential was performed using a custom MATLAB script (MathWorks).

Statistics. All data were subjected to statistical analysis in GraphPad Prism version 7. IL and PL groups were separate cohorts, and thus controls were not pooled; IL controls were compared to IL SSFO, while PL controls were compared to PL SSFO. When SSFO-stimulated VIP mice were compared to nonstimu- 
lated controls, 2-tailed, unpaired $t$ tests were performed (Figures 1-4). For multiple HFD exposure assays, mice received the same treatments across multiple exposures, and data were analyzed with 2-way repeated measures ANOVA and Tukey's post hoc comparisons with treatment and time points as independent variables (Figure 5, A-D). Mice served as their own controls in the operant responding experiment, and thus a 2-way repeated measures ANOVA was used (Figure 4D). For the 24-hour HCD2 consumption study, linear regression models were created with time and treatment group as fixed effects to determine whether differences between control and experimental animals were statistically significant. Separate models were created for light hours (0-12) and dark hours (13-24) (Figure 6, E and F). Data from once-performed behavioral tests (open field, elevated plus maze, novel object, social interaction) were analyzed with unpaired, 2-tailed $t$ tests (Figure 7 and Figure 8). All Data are expressed as mean \pm SEM. Statistical significance was considered when $P$ values were less than 0.05 . For the comparison between FR completion times, we used multiple $t$ tests with correction for multiple testing, using the Holm-Šídák method with $\alpha=5 \%$. For the c-Fos analysis, cell numbers and percentages of double-labeled cells were analyzed using 2-tailed, unpaired $t$ tests. In all bar graphs and tables, all grouped values are expressed as means and SEM.

\section{Study approval}

All experimental procedures were conducted in accordance with NIH guidelines and approved by the University of Virginia's Animal Care and Usage Committee (Charlottesville, Virginia, USA).

\section{Author contributions}

BAN designed experiments, performed all animal surgeries, conducted experiments, analyzed data, designed and constructed figures, and wrote the manuscript. CMW conducted experiments and constructed figures. PMK conducted electrophysiological experiments. MPB conducted electrophysiological experiments and analyzed subsequent data. MKJ conducted statistical analyses for extended food intake studies. MMS designed experiments, analyzed data, constructed figures, co-wrote the manuscript.

\section{Acknowledgments}

The authors would like to acknowledge Ruth L. Stornetta for her assistance with microscopy. This project was funded by a research grant from the NIH to MMS (1R01MH116694-01), while BAN received support from an NIH training grant (T32 GM007055)

Address correspondence to: Michael M. Scott, PO Box 800735, 1340 Jefferson Park Avenue, Pinn Hall, Room 5050, Charlottesville, Virgina 22908, USA. Phone: 434.243.1920; Email: michael.scott@virginia.edu.

1. Johnson PM, Kenny PJ. Dopamine D2 receptors in addiction-like reward dysfunction and compulsive eating in obese rats. Nat Neurosci. 2010;13(5):635-641.

2. Volkow ND, Wise RA, Baler R. The dopamine motive system: implications for drug and food addiction. Nat Rev Neurosci. 2017;18(12):741-752.

3. Liang B, et al. Distinct and dynamic ON and OFF neural ensembles in the prefrontal cortex code social exploration. Neuron. 2018;100(3):700-714.e9.

4. Corwin RL, et al. Binge-type eating disrupts dopaminergic and GABAergic signaling in the prefrontal cortex and ventral tegmental area. Obesity (Silver Spring). 2016;24(10):2118-2125.

5. Richard JM, Berridge KC. Prefrontal cortex modulates desire and dread generated by nucleus accumbens glutamate disruption. Biol Psychiatry. 2013;73(4):360-370.

6. Matsumoto M, Matsumoto K, Abe H, Tanaka K. Medial prefrontal cell activity signaling prediction errors of action values. Nat Neurosci. 2007;10(5):647-656.

7. Pi HJ, Hangya B, Kvitsiani D, Sanders JI, Huang ZJ, Kepecs A. Cortical interneurons that specialize in disinhibitory control. Nature. 2013;503(7477):521-524.

8. Seo DO, et al. A GABAergic projection from the centromedial nuclei of the amygdala to ventromedial prefrontal cortex modulates reward behavior. J Neurosci. 2016;36(42):10831-10842.

9. Ventura R, Morrone C, Puglisi-Allegra S. Prefrontal/accumbal catecholamine system determines motivational salience attribution to both reward- and aversion-related stimuli. Proc Natl Acad Sci U S A. 2007;104(12):5181-5186.

10. Warthen DM, et al. Activation of pyramidal neurons in mouse medial prefrontal cortex enhances food-seeking behavior while reducing impulsivity in the absence of an effect on food intake. Front Behav Neurosci. 2016;10:63.

11. Land BB, et al. Medial prefrontal D1 dopamine neurons control food intake. Nat Neurosci. 2014;17(2):248-253.

12. Mena JD, Selleck RA, Baldo BA. Mu-opioid stimulation in rat prefrontal cortex engages hypothalamic orexin/hypocretin-containing neurons, and reveals dissociable roles of nucleus accumbens and hypothalamus in cortically driven feeding. $J$ Neurosci. 2013;33(47):18540-18552. 
13. Mena JD, Sadeghian K, Baldo BA. Induction of hyperphagia and carbohydrate intake by $\mu$-opioid receptor stimulation in circumscribed regions of frontal cortex. J Neurosci. 2011;31(9):3249-3260.

14. Blasio A, Steardo L, Sabino V, Cottone P. Opioid system in the medial prefrontal cortex mediates binge-like eating. Addict Biol. 2014;19(4):652-662.

15. Moorman DE, Aston-Jones G. Prefrontal neurons encode context-based response execution and inhibition in reward seeking and extinction. Proc Natl Acad Sci U S A. 2015;112(30):9472-9477.

16. Peters J, LaLumiere RT, Kalivas PW. Infralimbic prefrontal cortex is responsible for inhibiting cocaine seeking in extinguished rats. J Neurosci. 2008;28(23):6046-6053.

17. Martín-García E, et al. Frequency of cocaine self-administration influences drug seeking in the rat: optogenetic evidence for a role of the prelimbic cortex. Neuropsychopharmacology. 2014;39(10):2317-2330.

18. Wall NR, De La Parra M, Sorokin JM, Taniguchi H, Huang ZJ, Callaway EM. Brain-wide maps of synaptic input to cortical interneurons. J Neurosci. 2016;36(14):4000-4009.

19. Rudy B, Fishell G, Lee S, Hjerling-Leffler J. Three groups of interneurons account for nearly $100 \%$ of neocortical GABAergic neurons. Dev Neurobiol. 2011;71(1):45-61.

20. Bourgeois JP, et al. Modulation of the mouse prefrontal cortex activation by neuronal nicotinic receptors during novelty exploration but not by exploration of a familiar environment. Cereb Cortex. 2012;22(5):1007-1015.

21. Alitto HJ, Dan Y. Cell-type-specific modulation of neocortical activity by basal forebrain input. Front Syst Neurosci. $2012 ; 6: 79$.

22. Pinto L, Dan Y. Cell-type-specific activity in prefrontal cortex during goal-directed behavior. Neuron. 2015;87(2):437-450.

23. Yizhar $\mathrm{O}$, et al. Neocortical excitation/inhibition balance in information processing and social dysfunction. Nature. 2011;477(7363):171-178.

24. Kawaguchi Y, Kubota Y. Physiological and morphological identification of somatostatin- or vasoactive intestinal polypeptide-containing cells among GABAergic cell subtypes in rat frontal cortex. J Neurosci. 1996;16(8):2701-2715.

25. Richard JM, Berridge KC. Prefrontal cortex modulates desire and dread generated by nucleus accumbens glutamate disruption. Biol Psychiatry. 2013;73(4):360-370.

26. Beckmann JS, Marusich JA, Gipson CD, Bardo MT. Novelty seeking, incentive salience and acquisition of cocaine self-administration in the rat. Behav Brain Res. 2011;216(1):159-165.

27. Hansson C, et al. Ghrelin influences novelty seeking behavior in rodents and men. PLoS One. 2012;7(12):e50409.

28. Panaro BL, Cone RD. Melanocortin-4 receptor mutations paradoxically reduce preference for palatable foods. Proc Natl Acad Sci U S A. 2013;110(17):7050-7055

29. Rolls ET, Murzi E, Yaxley S, Thorpe SJ, Simpson SJ. Sensory-specific satiety: food-specific reduction in responsiveness of ventral forebrain neurons after feeding in the monkey. Brain Res. 1986;368(1):79-86.

30. Reichelt AC, Morris MJ, Westbrook RF. Cafeteria diet impairs expression of sensory-specific satiety and stimulus-outcome learning. Front Psychol. 2014;5:852.

31. Hershenberg R, et al. Diminished effort on a progressive ratio task in both unipolar and bipolar depression. $J$ Affect Disord. 2016;196:97-100.

32. Bryce CA, Floresco SB. Perturbations in effort-related decision-making driven by acute stress and corticotropin-releasing factor. Neuropsychopharmacology. 2016;41(8):2147-2159.

33. Bergamini G, et al. Mouse psychosocial stress reduces motivation and cognitive function in operant reward tests: A model for reward pathology with effects of agomelatine. Eur Neuropsychopharmacol. 2016;26(9):1448-1464.

34. Vialou V, et al. Prefrontal cortical circuit for depression- and anxiety-related behaviors mediated by cholecystokinin: role of DeltaFosB. J Neurosci. 2014;34(11):3878-3887.

35. Bi LL, et al. Enhanced excitability in the infralimbic cortex produces anxiety-like behaviors. Neuropharmacology. 2013;72:148-156.

36. Elmquist JK, Coppari R, Balthasar N, Ichinose M, Lowell BB. Identifying hypothalamic pathways controlling food intake, body weight, and glucose homeostasis. J Comp Neurol. 2005;493(1):63-71.

37. Krashes MJ, et al. Rapid, reversible activation of AgRP neurons drives feeding behavior in mice. J Clin Invest. 2011;121(4):1424-1428.

38. Liu J, et al. Enhanced AMPA receptor trafficking mediates the anorexigenic effect of endogenous glucagon-like peptide-1 in the paraventricular hypothalamus. Neuron. 2017;96(4):897-909.e5.

39. Radley JJ, Gosselink KL, Sawchenko PE. A discrete GABAergic relay mediates medial prefrontal cortical inhibition of the neuroendocrine stress response. J Neurosci. 2009;29(22):7330-7340.

40. Murugan M, et al. Combined social and spatial coding in a descending projection from the prefrontal cortex. Cell. 2017;171(7):1663-1677.e16.

41. Mannella F, Gurney K, Baldassarre G. The nucleus accumbens as a nexus between values and goals in goal-directed behavior: a review and a new hypothesis. Front Behav Neurosci. 2013;7:135.

42. Munuera J, Rigotti M, Salzman CD. Shared neural coding for social hierarchy and reward value in primate amygdala. Nat Neurosci. 2018;21(3):415-423.

43. Maldonado-Irizarry CS, Swanson CJ, Kelley AE. Glutamate receptors in the nucleus accumbens shell control feeding behavior via the lateral hypothalamus. The Journal of neuroscience: the official journal of the Society for Neuroscience. 1995;15(10):6779-6788.

44. Vertes RP. Differential projections of the infralimbic and prelimbic cortex in the rat. Synapse. 2004;51(1):32-58.

45. Gabbott PL, Warner TA, Jays PR, Salway P, Busby SJ. Prefrontal cortex in the rat: projections to subcortical autonomic, motor, and limbic centers. J Comp Neurol. 2005;492(2):145-177.

46. Zhang Y, et al. Leptin-receptor-expressing neurons in the dorsomedial hypothalamus and median preoptic area regulate sympathetic brown adipose tissue circuits. J Neurosci. 2011;31(5):1873-1884.

47. Jeong JH, Lee DK, Jo YH. Cholinergic neurons in the dorsomedial hypothalamus regulate food intake. Mol Metab. 2017;6(3):306-312.

48. Marchant NJ, Furlong TM, McNally GP. Medial dorsal hypothalamus mediates the inhibition of reward seeking after extinction. J Neurosci. 2010;30(42):14102-14115.

49. Walker F, et al. Parvalbumin- and vasoactive intestinal polypeptide-expressing neocortical interneurons impose differential inhi- 
bition on Martinotti cells. Nat Commun. 2016;7:13664.

50. Karnani MM, et al. Cooperative subnetworks of molecularly similar interneurons in mouse neocortex. Neuron. 2016;90(1):86-100.

51. Zhou X, Rickmann M, Hafner G, Staiger JF. Subcellular targeting of VIP boutons in mouse barrel cortex is layer-dependent and not restricted to interneurons. Cereb Cortex. 2017;27(11):5353-5368.

52. Kim H, Ährlund-Richter S, Wang X, Deisseroth K, Carlén M. Prefrontal parvalbumin neurons in control of attention. Cell. 2016;164(1-2):208-218.

53. Vogt BA, Paxinos G. Cytoarchitecture of mouse and rat cingulate cortex with human homologies. Brain Struct Funct. 2014;219(1):185-192.

54. Xiong B, et al. Precise cerebral vascular atlas in stereotaxic coordinates of whole mouse brain. Front Neuroanat. 2017;11:128.

55. Gaykema RP, et al. Characterization of excitatory and inhibitory neuron activation in the mouse medial prefrontal cortex following palatable food ingestion and food driven exploratory behavior. Front Neuroanat. 2014;8:60.

56. Golden SA, Covington HE, Berton O, Russo SJ. A standardized protocol for repeated social defeat stress in mice. Nat Protoc. 2011;6(8):1183-1191.

57. Scott MM, et al. Hcrtr1 and 2 signaling differentially regulates depression-like behaviors. Behav Brain Res. 2011;222(2):289-294.

58. Klein PM, Lu AC, Harper ME, McKown HM, Morgan JD, Beenhakker MP. Tenuous inhibitory GABAergic signaling in the reticular thalamus. J Neurosci. 2018;38(5):1232-1248. 